\title{
Altered microRNA profile during fracture healing in rats with diabetes
}

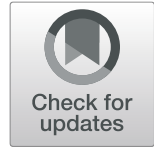

Shunsuke Takahara ${ }^{1,2+}$, Sang Yang Lee ${ }^{1,3 \dagger}$, Takashi Iwakura ${ }^{1}$, Keisuke Oe ${ }^{1}$, Tomoaki Fukui ${ }^{1}$, Etsuko Okumachi ${ }^{1}$, Michio Arakura', Yoshitada Sakai ${ }^{4}$, Tomoyuki Matsumoto' ${ }^{1}$, Takehiko Matsushita', Ryosuke Kuroda ${ }^{1}$ and Takahiro Niikura ${ }^{1 *}$

\begin{abstract}
Background: MicroRNAs (miRNAs) are a class of small non-coding RNA molecules that regulate gene expression. There is increasing evidence that some miRNAs are involved in the pathology of diabetes mellitus (DM) and its complications. We hypothesized that the functions of certain miRNAs and the changes in their patterns of expression may contribute to the pathogenesis of impaired fractures due to DM.

Methods: In this study, 108 male Sprague-Dawley rats were divided into DM and control groups. DM rats were created by a single intravenous injection of streptozotocin. Closed transverse femoral shaft fractures were created in both groups. On post-fracture days 5, 7, 11, 14, 21, and 28, miRNA was extracted from the newly generated tissue at the fracture site. Microarray analysis was conducted with miRNA samples from each group on post-fracture days 5 and 11. The microarray findings were validated by real-time polymerase chain reaction (PCR) analysis at each time point.
\end{abstract}

Results: Microarray analysis revealed that, on days 5 and 11, 368 and 207 miRNAs, respectively, were upregulated in the DM group, compared with the control group. The top four miRNAs on day 5 were miR-339-3p, miR451-5p, miR532-5p, and miR-551b-3p. The top four miRNAs on day 11 were miR-221-3p, miR376a-3p, miR-379-3p, and miR-3795p. Among these miRNAs, miR-221-3p, miR-339-3p, miR-376a-3p, miR-379-5p, and miR-451-5p were validated by real-time PCR analysis. Furthermore, PCR analysis revealed that these five miRNAs were differentially expressed with dynamic expression patterns during fracture healing in the DM group, compared with the control group.

Conclusions: Our findings will aid in understanding the pathology of impaired fracture healing in DM and may support the development of molecular therapies using miRNAs for the treatment of impaired fracture healing in patients with DM.

Keywords: Diabetes mellitus, MicroRNA, Fracture healing, Delayed union, Nonunion

\footnotetext{
* Correspondence: tniikura@med.kobe-u.ac.jp

†Shunsuke Takahara and Sang Yang Lee contributed equally to this work. 'Department of Orthopaedic Surgery, Kobe University Graduate School of Medicine, 7-5-1 Kusunoki-cho, Chuo-ku, Kobe 650-0017, Japan

Full list of author information is available at the end of the article
} 


\section{Background}

Diabetes mellitus (DM) is a major public health concern that is approaching epidemic proportions globally. There are 425 million people worldwide who have DM, and this number is expected to increase to 629 million by 2045 [1]. DM is associated with an extensive list of complications involving nearly every tissue in the body, including the heart, blood vessels, eyes, kidneys, and nerves. It has been reported that DM adversely affects bone health and is associated with reduced bone mineral density or reduced bone strength, resulting in a higher risk of fracture [2]. Clinical studies have shown higher incidences of delayed union and nonunion, as well as a doubling of the time to healing of fractures in patients with DM, compared with healthy patients $[3,4]$. In patients with DM, fracture healing time is prolonged by up to $87 \%$ [3]. In addition, Hernandez et al. reported higher odds of delayed union in patients with DM [4]. Despite reports of associations between DM and impaired fracture healing, including delayed union and nonunion, only a few investigations have examined the molecular mechanisms by which DM affects the process of fracture healing [5-7].

microRNAs (miRNAs) are short, single-stranded, noncoding RNA molecules that regulate gene expression [8]. miRNAs play pivotal roles in biological processes, including cellular differentiation, cell growth, and organ development [9]. In the field of skeletal biology, several studies showed that miRNAs contribute to the regulation of osteoblast, chondrocyte, and osteoclast differentiation and function, suggesting that miRNAs have important roles in bone formation, resorption, remodeling, and repair [10-12]. In addition, the involvement of miRNAs in fracture healing and nonunion has been demonstrated [10, 13-18].

Recently, some miRNAs were reported to be involved in the pathology of DM and its complications [19-21]. Chronic inflammation is an important determinant of insulin resistance and contributes to microvascular complications of type 2 DM, such as nephropathy, neuropathy, and retinopathy. miR-146a has been reported to regulate genes involved in the pathogenesis of type 2 DM and its related complications by attenuating inflammatory cytokine production in macrophages [20]. Grieco et al. reported hyperexpression of miR-148a and miR21-5p in serum/plasma from patients with type I DM. miR-148a and miR-21-5p target pivotal genes involved in bone development, modeling, and remodeling (e.g., wingless-type MMTV integration site family, member 1 (WNT1), and transforming growth factor- $\beta 2$ (TGF- $\beta 2$ )), thus suggesting that miR-148a and miR-21-5p serve as contributing factors to bone fragility or as potential biomarkers of bone metabolic alterations in patients with type 1 DM [21]. However, only a few studies have shown direct roles for miRNAs in impaired fracture healing in patients with DM or in animal models of DM [22].

The elucidation of miRNA expression changes during fracture healing may reveal valuable information regarding the pathophysiology of the healing process in patients with DM and suggests potential therapeutic interventions to accelerate fracture healing. This study was conducted to examine miRNA expression profiles during fracture healing of the femur of rats with DM by using microarray analysis and to elucidate the dynamic expression patterns of expressed miRNAs during fracture healing by using real-time polymerase chain reaction (PCR) analysis.

\section{Methods \\ Animals}

A total of one hundred and eight 10-week-old male Sprague-Dawley rats (SLC, Hamamatsu, Japan) were used in this study; 54 were assigned to the DM group and 54 to the control group. We created type I DM rats, as an impaired fracture healing model, by a single intraperitoneal injection of $40 \mathrm{mg} / \mathrm{kg}$ streptozotocin (STZ) (Sigma-Aldrich, St. Louis, MO, USA) [6, 7]. Rats with blood glucose levels over $300 \mathrm{mg} / \mathrm{dl}$ at 1 week after injection of STZ were used for this study as DM rats. Control rats with normal blood glucose levels were injected with sodium chloride as a sham treatment. All animal procedures were approved by the Animal Care and Use Committee of Kobe University Graduate School of Medicine (approval number: P131204).

\section{Surgical procedure}

Closed transverse femoral shaft fractures in both groups were produced as previously described, via insertion of a 1.25-mm-diameter Kirschner wire into the right femoral intramedullary canal and induction of a fracture using a three-point bending apparatus with a drop weight $[7,23]$. Preoperatively, we injected medetomidine $(0.15 \mathrm{mg} / \mathrm{kg})$, midazolam $(2 \mathrm{mg} / \mathrm{kg})$, and butorphanol $(2.5 \mathrm{mg} / \mathrm{kg})$ intraperitoneally for anesthesia and sedation. Unprotected weight bearing was allowed postoperatively. At each time points of assessment, all animals were anesthetized by isoflurane and euthanized by intraperitoneal injection of fatal dose pentobarbital sodium unconsciously.

\section{Radiographic assessment of fracture healing}

On post-fracture days $7,14,21$, and 28 , eight animals in each group were killed, and radiographs of the fracture site were acquired. Each callus on the four cortices (two from anteroposterior and two from lateral views) was evaluated by two orthopedic surgeons blinded to animal grouping. A bony union was defined when three of four 
cortices were bridged and/or fracture lines disappeared completely [24].

\section{Strategy for miRNA expression analysis}

Figure 1 shows the experimental design for miRNA expression analysis. As a first step, on post-fracture days 5 and 11, extracted miRNAs from the calluses from both groups were screened by microarray analysis for 727 miRNAs to identify those expressed at high levels in the DM group, compared with the control group. miRNAs were extracted by filtering with a fold change of $>2.0$ or coefficient variation $(<50 \%)$ and high Hy3 signal $(>10)$ $[13,14]$. Subsequently, these results were validated by real-time PCR analysis. This process was performed to compare the expression levels of selected miRNAs between the two groups at each time point and to investigate their changes in expression over time in each group.

\section{RNA extraction and miRNA microarray analysis}

On post-fracture days 5 and 11, five animals in each group were killed, and the fractured femurs were harvested. Total RNA, including miRNAs, was extracted from the newly generated calluses around the fracture site using the miRNeasy Mini Kit (Qiagen, Venlo, Netherlands). With the 3D-Gene miRNA Labeling Kit
(Toray, Kamakura, Japan), extracted total RNA was labeled. Labeled RNAs were hybridized onto 3D-Gene Rat miRNA Oligo chips (Toray). The annotation and oligonucleotide sequences of the probes were conformed in the miRBase miRNA database (http://www.mirbase.org/ ). After stringent washes, fluorescent signals were scanned with the 3D-Gene Scanner (Toray) and analyzed using the 3D-Gene Extraction software (Toray).

\section{Real-time PCR analysis}

Based on the array results, the top four miRNAs on days 5 and 11 were selected for further real-time PCR analysis. The newly generated calluses collected on postfracture days $5,7,11,14,21$, and 28 were harvested ( $n=$ 6 per group at each time point). The calluses were homogenized, and total RNA was extracted. RNA was reverse-transcribed to single-stranded cDNA using the miRCURY LNA Universal RT microRNA PCR Kit (Exiqon, Vedbaek, Denmark). Real-time PCR analysis was performed in duplicate with a StepOne Sequence Detector (Applied Biosystems, Branchburg, NJ, USA), using SYBR Green Master Mix and microRNA LNA PCR primer sets (both from Exiqon). As an internal control gene in miRNA PCR assays, U6 was used $[13,14]$. The conditions of the PCR amplification were $95^{\circ} \mathrm{C}$ for $30 \mathrm{sec}$, followed by 40 cycles of $95^{\circ} \mathrm{C}$ for $30 \mathrm{sec}$ and $75^{\circ} \mathrm{C}$ for

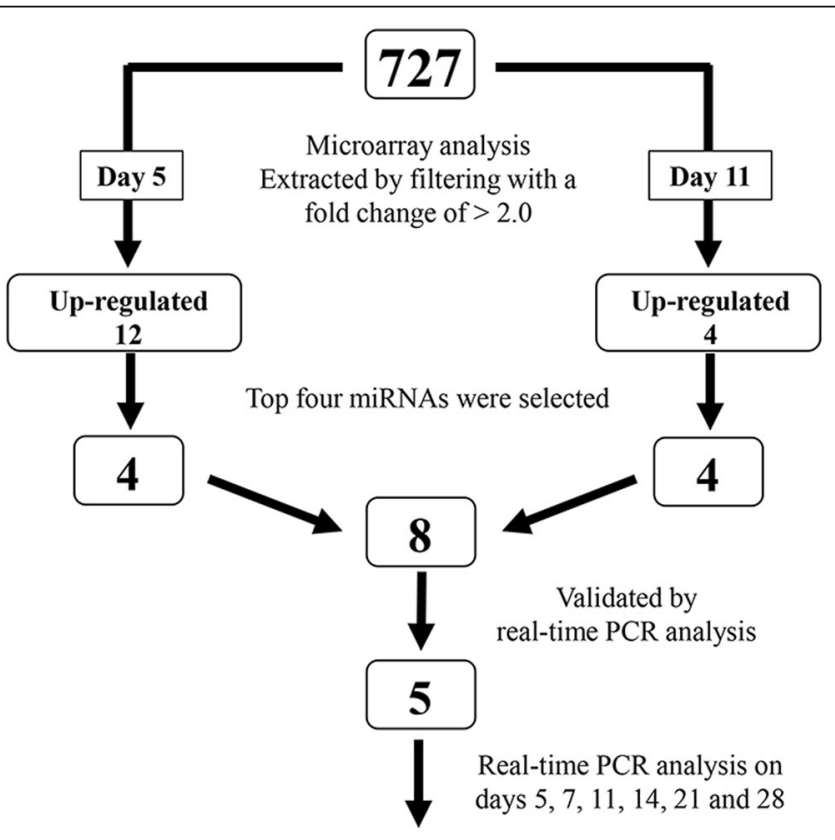

i) Comparison of the expression levels of the selected miRNAs between the two groups at each time point

ii) Evaluation of changes in expression of the selected miRNAs over time in each group

Fig. 1 Flow chart of the experimental design. The boxes indicate microRNA (miRNA) numbers; PCR, polymerase chain reaction 
$15 \mathrm{sec}$. Changes in miRNA expression levels were calculated using the comparative $\Delta \Delta C T$ method $[13,14]$ and is presented as the fold change relative to the level of the corresponding miRNA in the control group on postfracture day 5 .

\section{Statistical analysis}

All quantitative data are expressed as means \pm standard errors and were analyzed by the SPSS 18.0 software (SPSS Inc., Chicago, IL, USA). Fisher's exact test was used for radiographic assessment of the union rates between the groups at each time point. The values of the $\mathrm{DM}$ and control groups were compared at each time point using the Mann-Whitney $U$ test. The KruskalWallis test and Mann-Whitney $U$ test with Bonferroni correction were used to compare values among time points in each group. A $p$ value less than 0.05 was defined as statistically significant.

\section{Results}

\section{Radiographic assessment of fracture healing}

Table 1 presents the union rate by radiographic assessment. On day 21, enlargement of the callus was observed, and three (37.5\%) animals had achieved fracture union in the control group. On day 28 , in the control group, seven (87.5\%) of the animals had achieved union. On the other hands, there were no animals achieving union in the DM group on days 21 and 28. Moreover, remodeling processes were observed in the control group (Fig. 2).

\section{miRNA microarray analysis}

As presented in Fig. 1, using a miRNA-based array screening, we tested the expression levels of 727 rat miRNAs. On days 5 and 11, 368 and 207 miRNAs were upregulated in the DM group compared with the control group. Among the upregulated miRNAs, those that were highly upregulated (greater than twofold change) were extracted by filtering. Using this threshold, we identified 12 miRNAs that were highly upregulated on day 5 and four miRNAs that were highly upregulated on day 11, respectively. The top four miRNAs on day 5 were miR339-3p, miR451-5p, miR-532-5p, and miR-551b-3p (Table 2). The top four miRNAs on day 11 were miR221-3p, miR376a-3p, miR-379-3p, and miR-379-5p

Table 1 Union rate by radiographic assessment

\begin{tabular}{lll}
\hline & Control & DM \\
\hline Day 7 & $0 / 8(0 \%)$ & $0 / 8(0 \%)$ \\
Day 14 & $0 / 8(0 \%)$ & $0 / 8(0 \%)$ \\
Day 21 & $3 / 8(37.5 \%)$ & $0 / 8(0 \%)$ \\
Day 28 & $7 / 8(87.5 \%)$ & $0 / 8(0 \%)^{*}$ \\
\hline${ }^{*} p<0.05$ compared with the contro
\end{tabular}

${ }^{*} p<0.05$ compared with the control group
(Table 3). These data were deposited in the NCBI Gene Expression Omnibus with the accession no. GSE76365.

\section{Selection of five miRNAs for real-time PCR analysis}

Five of the eight miRNAs, namely, miR-221-3p, miR339-3p, miR-376a-3p, miR-379-5p, and miR-451-5p, could be validated by real-time PCR analysis. Figure 3 presents the expression levels of these miRNAs in both groups over time.

$m i R-221-3 p$. miR-221-3p is reportedly associated with the stromal cell-derived factor 1 (SDF-1)/CXC chemokine receptor 4 (CXCR4) pathway and angiogenesis (Table 3) $[28,30]$. The expression levels of miR-221-3p on days 5,7 , and 11 were significantly higher in the DM group than in the control group (Fig. 3a). In the control group, miR-221-3p expression peaked on day 14 and significantly decreased on day 21 . In contrast, miR-221-3p expression in the DM group peaked on day 11 and significantly decreased on day 14 . The expression level peaked earlier in the DM group than in the control group.

miR-339-3p. miR-339-3p is reportedly associated with inflammation (Table 2) [26]. The miR-339-3p expression level on day 5 was significantly higher in the DM group than in the control group, whereas the expression levels on days 14, 21, and 28 were significantly lower in the DM group than in the control group (Fig. $3 \mathrm{~b}$ ). In the control group, the miR-339-3p expression level remained unchanged from day 5 to day 11 and significantly decreased on day 14 . The expression level in the DM group peaked on day 5 and then declined over time.

miR-376a-3p and miR-379-5p. miR-376a-3p and miR$379-5 \mathrm{p}$ are associated with chondrogenesis (Table 3 ) $[27,29]$. The expression level of miR-376a-3p in the control group peaked on day 5 and then declined over time. The expression levels on days 11, 14, 21, and 28 were significantly higher in the DM group than in the control group (Fig. 3c). The miR-379-5p expression level at each time point was significantly higher in the DM group than in the control group. In the DM group, the expression level remained unchanged from day 5 to day 11 and then decreased (Fig. 3d).

miR-451-5p. miR-451-5p is associated with angiogenesis and inflammation (Table 2) [25, 31]. miR-451-5p expression levels on days 5,11 , and 21 were significantly higher in the DM group than in the control group (Fig. $3 e)$. In the control and DM groups, the expression level peaked on day 14 and significantly decreased on day 21 .

\section{Discussion}

Recently, miRNAs have emerged as key regulators in the complex process of fracture healing [13-15]. In addition, 


\section{Control}

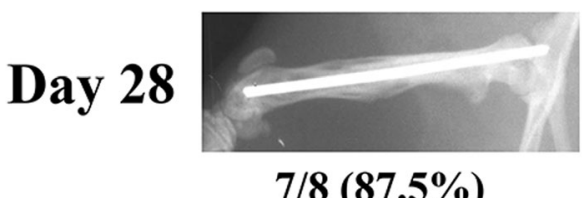

$7 / 8(87.5 \%)$

\section{DM}

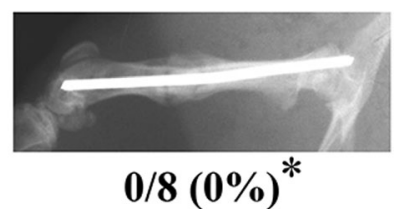

Fig. 2 Radiographs of femurs during fracture healing in the control and DM groups at day 28. Representative radiographs are shown, and the proportion of rats with fracture union is indicated at the bottom of images. ${ }^{*} p<0.05$ compared with the control group

some miRNAs are reportedly involved in the pathology of DM and its complications [19-21]. To better understand the underlying pathogenesis of impaired fracture healing in DM patients, we focused on miRNA expression profiles in fracture healing of the femur in diabetic rats. In the current study, we identified five miRNAs, miR-221-3p, miR-339-3p, miR-376a-3p, miR-379-5p, and miR-451-5p, which were expressed differentially with changing patterns during fracture healing in diabetic rats when compared with control rats. These findings provide new insights into the cellular processes involved in impaired fracture healing in patients with DM.

Bone fracture healing involves remarkably complex processes that require the coordination of a sequence of many biological events [32]. The rationale for the selection of two time points, post-fracture days 5 and 11, for microarray analysis was that key cellular events of the fracture healing process in healthy rats take place on days 5 and 11 . The process of fracture healing can be divided into three overlapping phases: inflammation, repair, and remodeling [32]. Post-fracture day 5 corresponds to the transitional period from the inflammatory phase to the repair phase [33]. Post-fracture day 11 corresponds to the repair phase, which involves the processes of intramembranous and endochondral ossifications, including osteogenesis, chondrogenesis, and vascular invasion [33]. Imbalance in inflammatory responses, reduced proliferation and differentiation of osteoblasts and chondrocytes, and reduced function and alteration in vascularization have been implicated as plausible pathogenic mechanisms underlying impaired fracture healing in patients with DM [2]. Previous

Table 2 Highly up-regulated miRNAs in DM group on postfracture day 5 and their validated putative genes

\begin{tabular}{lll}
\hline miRNA & Fold changeDM/control & Target genes \\
\hline miR-451-5p & 3.44 & $14-3-3 \zeta$ [25], Rab5a [25] \\
miR-532-5p & 3.23 & CXCL2 \\
miR-551b-3p & 3.04 & TMPRSS4 \\
miR-339-3p & 2.50 & ANXA3 [26]
\end{tabular}

CXC2L chemokine ligand 2, TMPRSS4 transmembrane protease serine 4, ANXA3 Annexin A3 studies showed that long-bone fractures of STZ-induced diabetic animals result in smaller calluses with reduced bone and cartilage formation, reduced proliferation and differentiation of osteoblasts and chondrocytes, and reduced mechanical strength $[6,7,34]$. In agreement with these previous studies, radiographic assessment in the present study showed that the fracture healing process in the DM group was delayed at days 21 and 28, compared with healing in the control group. Histological evaluation also revealed a delayed fracture healing process in the DM group, which was characterized by a smaller cartilage callus and a delay in endochondral ossification (Supplemental materials).

Inflammation is a critical factor during fracture healing, with inflammatory cells and molecular factors appearing locally at the fracture site in a distinct spatial and temporal manner [33]. Highly regulated inflammatory signaling during fracture healing is essential for priming bone regeneration. Disturbances in the finely tuned inflammatory responses at the fracture site lead to impaired vascularization, reduced bone formation, disturbed osteoclastic function, and subsequent delayed fracture healing or nonunion [35]. miR-339-3p was reported to inactivate the Akt/mammalian target of rapamycin (mTOR) signaling pathway and to alleviate inflammation and edema caused by severe acute pancreatitis with acute lung injury in a mouse model by targeting annexin A3 (ANXA3) [26]. In addition, rapamycin, an inhibitor of $\mathrm{mTOR}$, inhibits osteogenesis both in vitro and in vivo [36]. Inhibition of mTOR by rapamycin blocked insulin-like growth factor 1-induced osteoblast differentiation of mesenchymal stem cells and mineralization [36]. miR-451-3p reduces inflammation

Table 3 Highly upregulated miRNAs in DM group on postfracture day 11 and their putative target genes

\begin{tabular}{lll}
\hline miRNA & Fold change DM/control & Target genes \\
\hline miR-379-3p & 2.68 & Unknown \\
miR-376a-3p & 2.09 & BMPR2 [27], TGFBR1 [27] \\
miR-221-3p & 2.03 & SDF-1 [25], c-kit [28] \\
miR-379-5p & 2.02 & ATF3 [29] \\
\hline
\end{tabular}

BMPR2 bone morphogenetic protein receptor 2, TGFBR1 transforming growth factor beta receptor $1, S D F-1$ stromal cell derived factor-1 


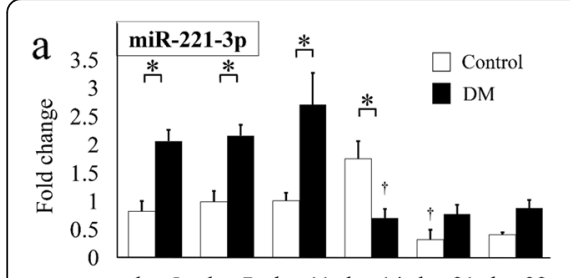

day 5 day 7 day 11 day 14 day 21 day 28
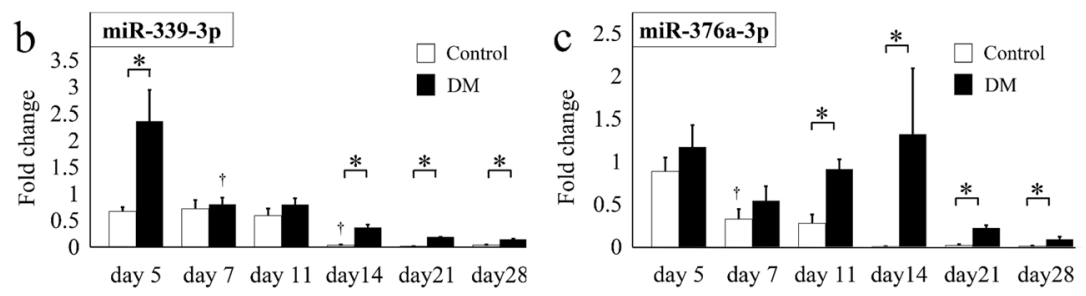
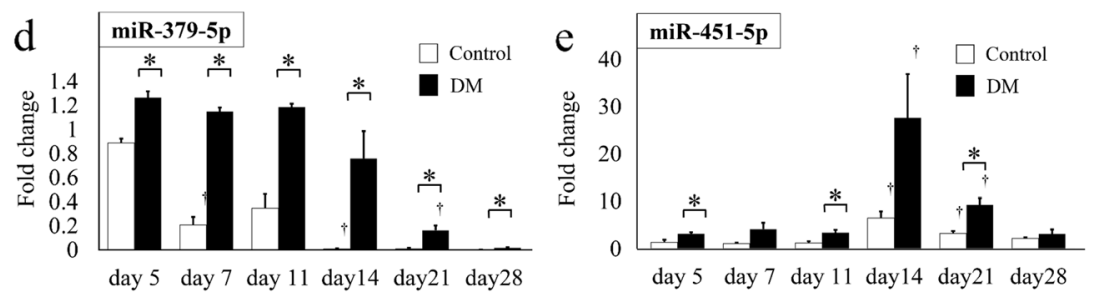

Fig. 3 Expression of selected miRNAs, miR-221-3p (a), miR-339-3p (b), miR-376a-3p (c), miR-379-5p (d), and miR-451-5p (e), in the control group (blank bars) and DM group (solid bars) on post-fracture days 5, 7, 11, 14, 21, and 28 as analyzed by real-time PCR. All graphs show the fold change in expression when the expression in the control group on day 5 was normalized as 1 . Values are the mean \pm standard error $(n=6$ in each group at each time point). ${ }^{*} p<0.05$ for indicated groups at the same time point.,$+ p<0.05$ versus values on the former time point in the same group

by suppressing phosphorylation of p38 mitogenactivated protein kinase (MAPK) via 14-3-3 $\zeta$ and Rab5a [25]. p38 MAPK plays an important signaling role in orchestrating injury or stress-induced responses, as well as in bone formation [37]. Pro-inflammatory cytokines, such as tumor necrosis factor- $\alpha$ and interleukin (IL)-1a, activate p38 MAPK; activation and signaling of p38 MAPK also lead to the production of these proinflammatory cytokines and their signal transduction. The interactions between p38 MAPK and proinflammatory cytokines are important for controlling life and death signaling cascades in osteoblasts and chondrocytes. Taken together, miR-339-3p and miR-451-3p may play important roles during fracture healing by regulating inflammation.

Chondrogenesis is an essential component of endochondral ossification in fracture healing [32]. miR-376a$3 \mathrm{p}$ is one of the most important miRNAs to trigger chondrogenesis [27]. Bakhshandeh et al. [27] reported that the expression of miR-376a was downregulated during the early phases of chondrogenic differentiation in unrestricted somatic stem cells. Bone morphogenetic protein (BMP) receptor 2 and TGF- $\beta$ receptor 1 are putative target genes of miR-376a [27]. Many studies have demonstrated that BMP-2 induces chondrogenic differentiation in various types of stem cell [38]. The recent study revealed that BMP- 2 and TGF- $\beta 1$ cooperate during chondrogenic differentiation of synovium-derived mesenchymal stem cells [39]. In addition, miR-379-5p, which is predicted to target activating transcription factor 3 (ATF3), regulates the endochondral ossification process by modulating proliferation and hypertrophic differentiation of the growth plate in male rats [29].
James et al. demonstrated the importance of ATF3 in the control of endochondral bone growth and skeletal development [40]. Some fundamental aspects of fracture healing, particularly endochondral ossification, have clear parallels with long bone development [41]. The altered expression of miR-376a-3p and miR-379-5p at the fracture site in rats with DM might dysregulate chondrogenesis and thus inhibit endochondral ossification, which could lead to impaired fracture healing.

Angiogenesis and bone formation are coupled during fracture healing [42]. A lack of oxygen (hypoxia) in fractured bone and the subsequent generation of angiogenic factors are critical for achieving successful fracture healing [43]. miR-451-5p was reported to inhibit cell growth, migration, and angiogenesis via downregulation of IL-6R [31]. miR-221-3p regulates angiogenesis by directly reducing the levels of c-Kit [28]. c-Kit and its ligand, stem cell factor (SCF), play an important role in the proliferation and differentiation of hematopoietic progenitor cells [44]. In addition, miR-221-3p inhibits the SDF-1/CXCR4 signaling pathway in human chondrocytes [30]. The SDF-1/CXCR4 axis plays a pivotal role in fracture healing by affecting the migration and differentiation of progenitor cells at fracture sites [7, 45, 46]. Kitaori et al. [45] reported that SDF-1 was induced in fractured bone and promoted endochondral bone formation by recruiting mesenchymal progenitor cells to the site of injury. Moreover, the SDF-1/CXCR4 axis acts as a key regulator of angiogenesis and contributes to the regulation of endothelial progenitor cell (EPC) recruitment in ischemic tissues. EPCs represent a small population of blood cells that can differentiate into endothelial cells and participate in postnatal angiogenesis [47]. In addition, EPCs 
are involved in physiological and pathological angiogenesis/vasculogenesis [48]. Recently, Arakura et al. [7] revealed that gene expression levels and localizations of SDF-1 and CXCR4 at fracture sites were altered during fracture healing in animals with experimental DM, which may contribute to the impaired fracture healing associated with inhibition of endochondral ossification and angiogenesis. Taken together, the altered expression patterns of miR-221-3p and miR-451-5p in the DM group during fracture healing suggest that disturbed angiogenesis/vasculogenesis and endochondral ossification may occur at fracture sites, potentially leading to impaired fracture healing.

The present results have clinical implications. Due to the increasing number of patients with DM, the incidence of impaired fracture healing associated with DM is expected to increase. It is desirable to establish strategies to prevent impaired fracture healing and enhance bone healing in patients with DM. Many biological and biophysical interventions have been attempted to accelerate fracture healing in these patients, including the use of exogenous growth factors, such as bone morphogenetic proteins, low-intensity pulsed ultrasound, and stem/progenitor cell transplantation [49]. Although most of these strategies have shown relatively satisfactory results, there are some notable limitations to their effectiveness and availability. The recent discovery of miRNAs, being powerful regulators in a variety of tissues and processes, suggests their potential as therapeutic target. Several therapeutic trials targeting miRNAs have been conducted [50].

Notably, the use of miravirsen (Santaris Pharma A/S, Copenhagen, Denmark), an anti-miR-122 drug, in patients with chronic hepatitis C (HCV) infection showed prolonged dose-dependent reductions in HCV RNA levels without evidence of viral resistance [51], which implies that miRNA-based therapeutics can indeed become a reality in clinical medicine. In the field of orthopedics, Murata et al. demonstrated that inhibition of miR-92a enhanced fracture healing in mice [15]. The five miRNAs identified in our study might represent a tool for the establishment of therapeutic approaches, which either prevent impaired fracture healing or enhance fracture healing. Further in vivo functional analyses will be needed to clarify the exact role and therapeutic potential of each miRNA during fracture healing.

This study had a few limitations. First, we did not demonstrate specific regulatory activities exerted by these five miRNAs; hence, our results solely indicate associations with impaired fracture healing, rather than clear causative relationships. Further in vivo analyses, including the loss or gain of miRNA function tests, are needed to define the exact role of each miRNA during fracture healing. Second, our study did not validate target genes of the five selected miRNAs. Target identification of miRNAs is computationally difficult due to the relatively low homology between miRNAs and their targets. Further, miRNAs may target any of the listed target genes in one cell type but not in others (given the diversity of cell types found within the fracture callus), and as such many may not be related specifically to bone or cartilage cells [17]. Further investigation is needed to clarify the contributions of each miRNA identified in this study to the downregulation of specific genes in the context of fracture healing process.

\section{Conclusions}

Altered expression levels of five miRNAs-miR-221-3p, miR-339-3p, miR-376a-3p, miR-379-5p, and miR-451$5 p-$ may contribute to impaired fracture healing in patients with DM. These findings provide valuable information to understand the pathology of impaired fracture healing in patients with DM, and may lead to the development of therapeutic strategies using miRNA for the treatment of fractures in patients with DM.

\section{Supplementary information}

Supplementary information accompanies this paper at https://doi.org/10. 1186/s13018-020-01658-X.

Additional file 1:. Figure $\mathrm{S1}$

Additional file 2:. Figure $\mathrm{S} 2$

\section{Abbreviations}

DM: Diabetes mellitus; miRNA: MicroRNA; mRNA: Messenger RNA; WNT1: Wingless-type MMTV integration site family, member 1; TGF$\beta 2$ : Transforming growth factor- $\beta 2$; STZ: Streptozotocin; PCR: Polymerase chain reaction; GEO: Gene Expression Omnibus; SDF-1: Stromal cell-derived factor 1; CXCR4: CXC chemokine receptor 4; mTOR: Mammalian target of rapamycin; ANXA3: Annexin A3; MAPK: Mitogen-activated protein kinase; IL: Interleukin; BMP: Bone morphogenetic protein; ATF3: Activating transcription factor 3; SCF: Stem cell factor; EPC: Endothelial progenitor cell

\section{Acknowledgements}

The authors would like to give a special thanks to Ms. M. Yasuda, Ms. K Tanaka, and Ms. M. Nagata (Department of Orthopaedic Surgery, Kobe University Graduate School of Medicine) for their excellent technical assistance.

\section{Authors' contributions}

ST: Study conception and design, acquisition of data, analysis and interpretation of data, drafting and revising the article. SL: Study conception and design, analysis and interpretation of data, drafting and revising the article. TI: Analysis and interpretation of data. KO: Analysis and interpretation of data, drafting and revising the article. TF: analysis and interpretation of data, drafting and revising the article. EO and MA: Acquisition of data. YS, TM1, TM2, and RK: Analysis and interpretation of data, drafting and revising the article. TN: Study conception and design, analysis and interpretation of data, drafting and revising the article. All authors read and approved the final manuscript.

\section{Funding}

This work was supported by a Grant-in-Aid for Scientific Research (C) from Japan Society for the Promotion of Science (15 K10443 to KO), and grants from the General Insurance Association in Japan. The funding sources had 
no role in the study design, data collection and analysis, decision to publish, or preparation of the manuscript.

\section{Availability of data and materials}

The datasets used and analyzed during the current study are available from the corresponding author on reasonable request.

\section{Ethics approval and consent to participate}

All animal procedures were performed under the approval and guidance of the Animal Care and Use Committee of Kobe University Graduate School of Medicine (approval number: P131204).

\section{Consent for publication}

Not applicable.

\section{Competing interests}

The authors declare that they have no competing interests.

\section{Author details}

'Department of Orthopaedic Surgery, Kobe University Graduate School of Medicine, 7-5-1 Kusunoki-cho, Chuo-ku, Kobe 650-0017, Japan. ²Department of Orthopaedic Surgery, Hyogo Prefectural Kakogawa Medical Center, Kakogawa 675-8555, Japan. ${ }^{3}$ Department of Orthopaedic Surgery, Showa University School of Medicine, Tokyo 142-8666, Japan. ${ }^{4}$ Division of Rehabilitation Medicine, Kobe University Graduate School of Medicine, Kobe 650-0017, Japan

\section{Received: 18 February 2020 Accepted: 27 March 2020}

\section{Published online: 07 April 2020}

\section{References}

1. No author listed. Na. IDF diabetes atlas - 8th edition. http://diabetesatlas.org (data last ).

2. Jiao $H$, Xiao E, Graves DT. Diabetes and its effect on bone and fracture healing. Curr Osteoporos Rep. 2015;13:327-35.

3. Loder RT. The influence of diabetes mellitus on the healing of closed fractures. Clin Orthop Relat Res. 1988:210-6.

4. Hernandez RK, Do TP, Critchlow CW, Dent RE, Jick SS. Patient-related risk factors for fracture-healing complications in the United Kingdom general practice research database. Acta Orthop. 2012;83:653-60.

5. Gaston MS, Simpson AH. Inhibition of fracture healing. J Bone Joint Surg (Br). 2007;89:1553-60.

6. Ogasawara A, Nakajima A, Nakajima F, Goto K, Yamazaki M. Molecular basis for affected cartilage formation and bone union in fracture healing of the streptozotocin-induced diabetic rat. Bone. 2008;43:832-9.

7. Arakura M, Lee SY, Takahara S, Okumachi E, Iwakura T, Fukui T, et al. Altered expression of SDF-1 and CXCR4 during fracture healing in diabetes mellitus. Int Orthop. 2017:41:1211-7.

8. Bartel DP. MicroRNAs: genomics, biogenesis, mechanism, and function. Cell. 2004;116:281-97.

9. Zhang B, Wang Q, Pan X. MicroRNAs and their regulatory roles in animals and plants. J Cell Physiol. 2007;210:279-89.

10. Nugent M. MicroRNAs and fracture healing. Calcif Tissue Int. 2017;101:35561.

11. McAlinden A, Im Gl. MicroRNAs in orthopaedic research: disease associations, potential therapeutic applications, and perspectives. J Orthop Res. 2018:36:33-51.

12. Cao Y, Lv Q, Lv C. MicroRNA-153 suppresses the osteogenic differentiation of human mesenchymal stem cells by targeting bone morphogenetic protein receptor type II. Int J Mol Med. 2015;36:760-6.

13. Waki T, Lee SY, Niikura T, Iwakura T, Dogaki Y, Okumachi E, et al. Profiling microRNA expression in fracture nonunions: potential role of microRNAs in nonunion formation studied in a rat model. Bone Joint J. 2015;97-B:114451.

14. Waki T, Lee SY, Niikura T, Iwakura T, Dogaki Y, Okumachi E, et al. Profiling microRNA expression during fracture healing. BMC Musculoskelet Disord. 2016;17:83.

15. Murata K, Ito H, Yoshitomi H, Yamamoto K, Fukuda A, Yoshikawa J, et al. Inhibition of miR-92a enhances fracture healing via promoting angiogenesis in a model of stabilized fracture in young mice. J Bone Miner Res. 2014;29: $316-26$.
16. Sampson HW, Chaput CD, Brannen J, Probe RA, Guleria RS, Pan J, et al. Alcohol induced epigenetic perturbations during the inflammatory stage of fracture healing. Exp Biol Med (Maywood). 2011;236:1389-401.

17. Hadjiargyrou M, Zhi J, Komatsu DE. Identification of the microRNA transcriptome during the early phases of mammalian fracture repair. Bone. 2016;87:78-88.

18. Chen H, Ji X, She F, Gao Y, Tang P. miR-628-3p regulates osteoblast differentiation by targeting RUNX2: possible role in atrophic non-union. Int J Mol Med. 2017;39:279-86.

19. Pandey AK, Agarwal P, Kaur K, Datta M. MicroRNAs in diabetes: tiny players in big disease. Cell Physiol Biochem. 2009;23:221-32.

20. Alipoor B, Ghaedi H, Meshkani R, Torkamandi S, Saffari S, Iranpour M, et al. Association of miR-146a expression and type 2 diabetes mellitus: a metaanalysis. Int J Mol Cell Med. 2017;6:156-63.

21. Grieco GE, Cataldo D, Ceccarelli E, Nigi L, Catalano G, Brusco N, et al. Serum levels of miR-148a and miR-21-5p are increased in type 1 diabetic patients and correlated with markers of bone strength and metabolism. Noncoding RNA. 2018;4

22. Takahara S, Lee SY, Iwakura T, Oe K, Fukui T, Okumachi E, et al. Altered expression of microRNA during fracture healing in diabetic rats. Bone Joint Res. 2018:7:139-47.

23. Bonnarens F, Einhorn TA. Production of a standard closed fracture in laboratory animal bone. J Orthop Res. 1984;2:97-101.

24. Dijkman BG, Sprague $S$, Schemitsch EH, Bhandari M. When is a fracture healed? Radiographic and clinical criteria revisited. J Orthop Trauma. 2010; 24(Suppl 1):S76-80.

25. Murata $K$, Yoshitomi H, Furu M, Ishikawa M, Shibuya $H$, Ito $H$, et al. MicroRNA-451 down-regulates neutrophil chemotaxis via p38 MAPK. Arthritis Rheum. 2014;66:549-59.

26. Wu XM, Ji KQ, Wang HY, Zhao Y, Jia J, Gao XP, et al. MicroRNA-339-3p alleviates inflammation and edema and suppresses pulmonary microvascular endothelial cell apoptosis in mice with severe acute pancreatitis-associated acute lung injury by regulating Anxa3 via the Akt/ mTOR signaling pathway. J Cell Biochem. 2018;119:6704-14.

27. Bakhshandeh B, Soleimani M, Paylakhi SH, Ghaemi N. A microRNA signature associated with chondrogenic lineage commitment. J Genet. 2012:91:171 82

28. Poliseno L, Tuccoli A, Mariani L, Evangelista M, Citti L, Woods K, et al. MicroRNAs modulate the angiogenic properties of HUVECs. Blood. 2006;108: 3068-71.

29. Jee YH, Wang J, Yue S, Jennings M, Clokie SJH, Nilsson O, et al. Mir-374-5p, mir-379-5p, and mir-503-5p regulate proliferation and hypertrophic differentiation of growth plate chondrocytes in male rats. Endocrinology. 2018.

30. Zheng X, Zhao FC, Pang Y, Li DY, Yao SC, Sun SS, et al. Downregulation of miR-221-3p contributes to IL-1 beta-induced cartilage degradation by directly targeting the SDF1/CXCR4 signaling pathway. J Mol Med (Berl). 2017;95:615-27.

31. Liu D, Liu C, Wang X, Ingvarsson S, Chen H. MicroRNA-451 suppresses tumor cell growth by down-regulating IL6R gene expression. Cancer Epidemiol. 2014;38:85-92.

32. Dimitriou R, Tsiridis E, Giannoudis PV. Current concepts of molecular aspects of bone healing. Injury. 2005:36:1392-404

33. Rundle $\mathrm{CH}$, Wang H, Yu H, Chadwick RB, Davis El, Wergedal JE, et al. Microarray analysis of gene expression during the inflammation and endochondral bone formation stages of rat femur fracture repair. Bone. 2006;38:521-9.

34. Kasahara T, Imai S, Kojima H, Katagi M, Kimura H, Chan L, et al. Malfunction of bone marrow-derived osteoclasts and the delay of bone fracture healing in diabetic mice. Bone. 2010:47:617-25.

35. Claes $L$, Recknagel $S$, Ignatius $A$. Fracture healing under healthy and inflammatory conditions. Nat Rev Rheumatol. 2012;8:133-43.

36. Xian L, Wu X, Pang L, Lou M, Rosen CJ, Qiu T, et al. Matrix IGF-1 maintains bone mass by activation of mTOR in mesenchymal stem cells. Nat Med. 2012;18:1095-101.

37. Zhou FH, Foster BK, Zhou XF, Cowin AJ, Xian CJ. TNF-alpha mediates p38 MAP kinase activation and negatively regulates bone formation at the injured growth plate in rats. J Bone Miner Res. 2006;21:1075-88.

38. Zhou N, Li Q, Lin X, Hu N, Liao JY, Lin LB, et al. BMP2 induces chondrogenic differentiation, osteogenic differentiation and endochondral ossification in stem cells. Cell Tissue Res. 2016;366:101-11. 
39. Kovermann NJ, Basoli V, Della Bella E, Alini M, Lischer C, Schmal H, et al. BMP2 and TGF-beta cooperate differently during synovial-derived stem-cell chondrogenesis in a dexamethasone-dependent manner. Cells. 2019;8.

40. James CG, Woods A, Underhill TM, Beier F. The transcription factor ATF3 is upregulated during chondrocyte differentiation and represses cyclin D1 and A gene transcription. BMC Mol Biol. 2006;7:30.

41. Einhorn TA, Gerstenfeld LC. Fracture healing: mechanisms and interventions. Nat Rev Rheumatol. 2015;11:45-54.

42. Portal-Nunez S, Lozano D, Esbrit P. Role of angiogenesis on bone formation. Histol Histopathol. 2012;27:559-66.

43. Wang T, Wang Y, Menendez A, Fong C, Babey M, Tahimic CG, et al. Osteoblast-specific loss of IGF1R signaling results in impaired endochondral bone formation during fracture healing. J Bone Miner Res. 2015;30:1572-84.

44. Yamaguchi H, Ishii E, Saito S, Tashiro K, Fujita I, Yoshidomi S, et al. Umbilical vein endothelial cells are an important source of c-kit and stem cell factor which regulate the proliferation of haemopoietic progenitor cells. Br J Haematol. 1996;94:606-11.

45. Kitaori T, Ito H, Schwarz EM, Tsutsumi R, Yoshitomi H, Oishi S, et al. Stromal cell-derived factor $1 /$ CXCR4 signaling is critical for the recruitment of mesenchymal stem cells to the fracture site during skeletal repair in a mouse model. Arthritis Rheum. 2009;60:813-23.

46. Yellowley C. CXCL12/CXCR4 signaling and other recruitment and homing pathways in fracture repair. Bonekey Rep. 2013;2:300.

47. Kiewisz J, Kaczmarek MM, Pawlowska A, Kmiec Z, Stompor T. Endothelia progenitor cells participation in cardiovascular and kidney diseases: a systematic review. Acta Biochim Pol. 2016;63:475-82.

48. Watt SM, Athanassopoulos A, Harris AL, Tsaknakis G. Human endothelial stem/progenitor cells, angiogenic factors and vascular repair. J R Soc Interface. 2010;7(Suppl 6):S731-51.

49. Dimitriou $R$, Jones $E$, McGonagle D, Giannoudis PV. Bone regeneration: current concepts and future directions. BMC Med. 2011;9:66.

50. Soifer HS, Rossi JJ, Saetrom P. MicroRNAs in disease and potential therapeutic applications. Mol Ther. 2007:15:2070-9.

51. Janssen HL, Kauppinen S, Hodges MR. HCV infection and miravirsen. N Engl Jed. 2013;369:878

\section{Publisher's Note}

Springer Nature remains neutral with regard to jurisdictional claims in published maps and institutional affiliations.

Ready to submit your research? Choose BMC and benefit from:

- fast, convenient online submission

- thorough peer review by experienced researchers in your field

- rapid publication on acceptance

- support for research data, including large and complex data types

- gold Open Access which fosters wider collaboration and increased citations

- maximum visibility for your research: over $100 \mathrm{M}$ website views per year

At $\mathrm{BMC}$, research is always in progress.

Learn more biomedcentral.com/submissions 